\title{
KryptonEyed: Playing with Gaze Without Looking
}

\author{
Argenis Ramirez Gomez \\ Lancaster University \\ a.ramirezgomez@lancaster.ac.uk
}

\author{
Hans Gellersen \\ Aarhus University \\ hwg@cs.au.dk
}

\begin{abstract}
As eye-tracking technologies become more affordable, the number of mainstream gaze-enabled games increases. These allow triggering in-game actions when the eyes focus on objects and locations of interest. Such gaze interactions follow the interaction paradigm "what you look at is what you get". We challenge this use of gaze interaction and propose to play without looking - with the eyes closed. We designed the game prototype KryptonEyed to introduce closing the eyes for eyes-only game control. Players are required to close their eyes and perform eye movements behind the eyelids before opening them to aim the teleportation of the main character. The game contains three levels integrating the proposed gaze mechanic in distinct game scenarios. These explore different challenges in their game dynamics and interaction metaphors to use the technique in various contexts of play.
\end{abstract}

\section{CCS CONCEPTS}

- Human-centered computing $\rightarrow$ Human computer interaction (HCI).

\section{KEYWORDS}

Gaze Interaction, Games, Gaze Pointing, Game Design, Not Looking, Metaphors

\section{ACM Reference Format:}

Argenis Ramirez Gomez and Hans Gellersen. 2020. KryptonEyed: Playing with Gaze Without Looking. In International Conference on the Foundations of Digital Games (FDG '20), September 15-18, 2020, Bugibba, Malta. ACM, New York, NY, USA, 4 pages. https://doi.org/10.1145/3402942.3403017

\section{INTRODUCTION}

Eye-trackers have become a compelling and affordable mainstream technology targeting the PC gaming community [17]. In gazeenabled games, gaze interaction is aligned with looking at the object of interest [16], for instance, to shoot by looking at targets [5]; or to enable accessible game control [19]. Other work uses gaze more creatively exploring the social use of gaze, such as during staring contests [23]; or exploring social cues [21]. In sum, gaze-enabled games require the player to look at the game scene to interact with the eyes.

Permission to make digital or hard copies of all or part of this work for personal or classroom use is granted without fee provided that copies are not made or distributed for profit or commercial advantage and that copies bear this notice and the full citation on the first page. Copyrights for components of this work owned by others than ACM must be honored. Abstracting with credit is permitted. To copy otherwise, or republish, to post on servers or to redistribute to lists, requires prior specific permission and/or a fee. Request permissions from permissions@acm.org.

FDG '20, September 15-18, 2020, Bugibba, Malta

(C) 2020 Association for Computing Machinery.

ACM ISBN 978-1-4503-8807-8/20/09 . \$ \$15.00

https://doi.org/10.1145/3402942.3403017
In this work, we explore the contrary: how to use not looking playfully and the intentional closing of the eyelids to propose a new gaze interaction mechanic based on our ability to roll the eyes while closed. Moreover, we designed the game prototype KryptonEyed, including three different game dynamics, to explore novel game metaphors and mechanics using this gaze paradigm. In the game, we introduced the act of closing and opening the eyes to control the character's powers to teleport; attack; jump and rest. Our work contributes to gaze-enabled game design by proposing a novel gaze mechanic in use in a game prototype including three possible game dynamics. Future work can explore the potential of integrating gaze metaphors that include looking and not looking at the game in different game genres.

\section{RELATED WORK}

Eye-tracking has enabled the creation of a wide range of techniques that allow interacting with content with just looking at it. We can use our eyes for an implicit indication of interest [16] or explicit aim at targets to acquire them [6]. Overall, the eyes can perform different types of eye movements for interaction, namely, fixations [1]; saccades [24]; or smooth pursuits [22]. Moreover, eye movements can also happen with closed eyes, e.g. Rapid Eye Movement (REM) [7] while sleeping. Here, we investigated during the design of a game prototype how to integrate performing eye movements with the eyes closed as a gaze-enabled game mechanic.

Gaze has been used in a wide variety of ways in games [18]. The main thrust of eye interaction applications in games have used gaze for direct control of the avatars [15] or their tools and weapons; the game camera [9] or to adapt the game engine to the player's gaze behaviour [8]. Gaze is used as a natural pointer and the mechanism for selection of objects of interest. Our work is in contrast by proposing to play "without looking" and close the eyes for interaction.

"Not looking" is an unexpected gaze interaction in games [11]. Some examples have used this concept to make the users look away, e.g. as a response to social cues [21]; to deceive the opponent player [10]; because they are penalised [13]; or can't look at two events at once $[12,20]$. Here, we propose to leverage that we can still move our eyes when the eyelids are voluntary closed as a novel game mechanic, rather than prompting the user to look away from the scene.

\section{KRYPTONEYED}

KryptonEyed is a gaze-only controlled game that explores novel opportunities for gaze-enabled game design by requiring the closing of the eyes for interaction. The game introduces the closing and opening of the eyelids to make WanderEye, a superhero from unknown origins, teleport instantly across the game space. When players close their eyes, they can make WanderEye teleport to where they are looking once they open them. The objective of the game 

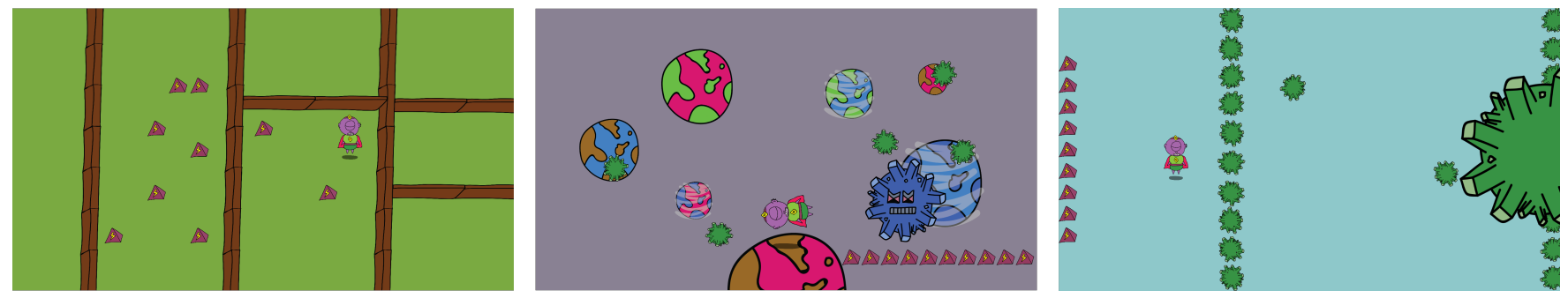

Figure 1: KryptonEyed game scenes in order of appearance. (Left) Introductory Maze level - Collection Puzzle Game Scene. (Center) Main task - Shooter Game Scene. (Right) Final Boss level - Fast-Paced Obstacle Jump Game.

is to help WanderEye battle and defeat the evil Krypto-Monsters while resisting the exposure to Kryptoneye - the hero's weakness that will make her lose all her energy, a dangerous material released by the monsters.

The game develops in three levels that explore the proposed gaze mechanic in three scenarios with different level of difficulty: Collection Puzzle (Introduction to the technique), Shooter Game (Main Task), and a Fast-Paced Obstacle Jump Game (Boss Level). All the levels use the gaze mechanic to teleport to allow WanderEye to navigate; attack; rest; and jump obstacles.

The game was developed using 2D custom graphics and creative commons sounds with Unity Game Engine using Tobii Gaming's SDK. The game requires the use of an eye-tracker and calibration is mandatory before playing. The game lasts between 2 and 3 minutes approximately.

\subsection{Collection Puzzle Game}

The Collection Puzzle level introduces the user to the game concept and gaze mechanic for interaction. The player needs to make WanderEye navigate the walled maze to collect the ten available energy boxes (see Figure 1, Left). In this level, WanderEye automatically moves towards the user's gaze point. Players can use her superpowers and make her teleport when a wall is encountered to move to the other side.

The level task is designed to have no challenge and help the player learn how to "teleport" to navigate the maze by closing and opening their eyes. Moreover, the level presents as an example of how the proposed technique could be combined with another gaze mechanic: "look to go there". This level aims to offer a relaxed and short experience that the users cannot fail to allow them to get used to controlling the main character.

\subsection{Shooter Game}

Once the first task is accomplished, the second level explores the potential of the interaction in a more challenging game dynamic: a shooter game. This second game investigates how well we can aim with gaze at targets that we do not see - with the eyes closed - playing with different types of eye movements that need to be performed.

In this level, WanderEye needs to save the galaxy by attacking the different Krypto-Monsters (see Figure 1, Center). WanderEye needs to hit 20 monsters to make them go away by teleporting herself on top of them, requiring the player to close their eyes and point at monsters before they open them to attack. Moreover, once the attack is performed, WanderEye gets back to her initial position.

Moreover, the galaxy is filled with Kryptoneye and will make WanderEye vulnerable. If players look around the scene, WanderEye will spin and lose energy. However, she can recover her powers by closing her eyes. This is introduced following the metaphor of closing your eyes to rest or "sleeping", implying that when using the teleportation powers to attack is a "tiring" event consuming energy that needs reloading. In the game, WanderEye has ten energy boxes, and each can be lost after two consecutive seconds looking at the scene. Accordingly, two seconds of rest charges one energy box, up to a maximum of ten. If the player loses all the power, the game is over and starts again from the previous level. This dynamic aimed to make the level more challenging by limiting the time players can look at the game and introduce the need to wait with their eyes closed to recharge and plan their next move.

Further, to explore different challenges, we designed three types of "Krypto-Monsters" that are inspired by three specific types of eye movements commonly used in gaze-based interaction: fixations; saccades and smooth pursuits. Each monster followed a set of rules and consequences to require players to perform different eye movements behind the eyelids. Kryptoxations are blue and static monsters that remain in the same position (based on gaze fixations). When looked at, they become smaller. Kryptoccades are red monsters based on saccadic motion. They move away to a different position every time they are hit or directly looked at, making it difficult to attack them unless the eyes are closed when players aim their gaze at them. Finally, Kryptosuits are yellow monsters that continuously move in an orbital loop based on smooth pursuit eye movements [2]. They are difficult to track because they speed up only when the player tries to look at them.

In design, the consequences of looking at the monsters target requiring to perform different eye behaviours with the eyes closed. For instance, Kryptoxations demand the player to swiftly close their eyes and maintain the same position, emulating a fixation. Conversely, the rules set for Kryptoccades and Kryptosuits aim to prevent the player from fixating at the monsters before performing the interactions, by moving them away from the gaze focus. In the game, all the monsters could appear anywhere in the screen and have between one and four lives - randomly set by the game engine - requiring to be attacked several times to disappear. Monsters' sizes varied randomly to increase the game challenge, from a diameter 
of 200px at the start, and 100px at the end. WanderEye size in the game is set too an area of $80 \mathrm{x} 80 \mathrm{px}$.

\subsection{Fast-Paced Obstacle Jump Game}

After defeating all the Krypto-Monsters, the player needs to defeat the last KryptoBoss. This last level explores the proposed gaze mechanic in a fast-paces game environment, emulating a jumping obstacles game.

In the last level, players need to help WanderEye teleport to perform quick jumps over Kryptoneye-made moving walls that the KryptoBoss is constantly firing by closing and opening their eyes. Moreover, the boss also fires single missiles made of the same dangerous material towards our hero (see Figure 1, Right). In this level, WanderEye is immune to look at Kryptoneye, but she needs to avoid the obstacles and wait for the right moment to attack the main monster. If she is hit by Kryptoneye, she will lose energy, which cannot be recharged when closing the eyes - under stressful events.

The player has the chance to attack the KryptoBoss if they are vulnerable, only when they are spinning. When WanderEye hits them, the big monster changes its position to the opposite side of the scene and carries on throwing Kryptoneye towards the hero. Kryptoneye is 70px diameter, whereas the boss is 350x250px size - half-hidden on the side of the scene. The game ends once the KryptoBoss is hit five times.

This level was designed to test the performance of the proposed gaze interaction at a more challenging and higher difficulty level in a fast-pace game dynamic. The main challenge is that interaction needs to be swift to avoid the constant stream of moving objects. Players need to be very familiar with how to use the technique and perform it quickly to succeed.

\section{DISCUSSION}

Gaze-enabled games are designed to leverage the natural pointing behaviour of the eyes, contributing to improving the players' performance. By doing this, designers might remove the challenge of the game as game objects are moved towards the gaze point [1], automatically tags enemies; or interact with objects is triggered at gaze. However, other games try to challenge these game mechanics by playing with our visual capabilities, e.g. attention dilemmas when players need to attend at two events at once [20]; or playing with peripheral vision [13].

KryptoneEyed is deliberately challenging and aims to use gaze to leverage the capabilities of our visual system. We require players to do something that is unexpected [11], closing the eyes and not look at the game graphics. This relies on spatial perception and the estimation of target locations in the game, but also memory by somewhat remembering the targeted area while recharging with the eyes closed. Moreover, the game relies on peripheral vision awareness by requiring players to look elsewhere before they close their eyes, e.g. to attack a Krypto-Monster. Overall, the game showcases new approaches to play with gaze not only when we look, but also leverage what we can do when we are not looking.

This paradigm is possible when combining the proposed gaze mechanic with metaphors that allow projecting behaviours from the real world to the game scene [14]. In KryptonEyed closing the eyes is contextualised with the metaphor of using a superpower: teleportation. However, the same paradigm is aligned with different behaviours such as moving (across the walls); to prepare an attack and sleep to recharge energy (shooter level), or disappear to jump an obstacle (Boss level).

Overall, KryptonEyed showcases new opportunities to design with gaze beyond looking metaphors by leveraging the inherent capabilities of the eyes and vision.

\subsection{Future work}

KryptonEyed showcases three game dynamics with different challenges, difficulty and stress levels and paces, commonly present in games with distinct phases such as the popular franchises Pokémon [3] and Zelda [4], e.g. adventure/exploration, battle, boss battle. However, the proposed gaze mechanic could be used in other game genres, and it should be evaluated in future work.

To integrate the closing of the eyes in game dynamics, designers can use metaphors as an asset to overcome the challenge of embedding this behaviour into the gameplay. Metaphors allow a diegesis between the interaction technique and the game story because they refer to a real phenomenon or behaviour with which the player can relate [14]; e.g. shut the eyes to rest or prepare an action. These allow the use of more realistic - possibly meaningful - applications of gaze beyond social cues [21] that leverage vision's capabilities and have the potential to increase the game's feeling of immersion.

Future work can address questions on the performance of the technique and its accuracy as well as further analyze the opportunities for games to integrate different paradigms of gaze interaction, including looking and not looking.

\section{CONCLUSION}

In this paper, we presented the game KryptonEyed exploring the use of the closing of the eyes to trigger gaze interaction as a novel gazeenabled game mechanic. The game illustrates three game scenarios that showcase how the presented mechanic could be embedded through the use of different design metaphors, for example, to teleport; to prepare an attack; recharge the hero's energy or jump. We discussed the potential of the mechanic in providing novel opportunities for gaze interaction in games.

\section{REFERENCES}

[1] Michael Dorr, Laura Pomarjanschi, and Erhardt Barth. 2009. Gaze beats mouse: A case study on a gaze-controlled breakout. PsychNology fournal 7, 2 (2009), 197-211.

[2] Augusto Esteves, Eduardo Velloso, Andreas Bulling, and Hans Gellersen. 2015. Orbits: Gaze interaction for smart watches using smooth pursuit eye movements. In Proceedings of the 28th Annual ACM Symposium on User Interface Software \& Technology. ACM, 457-466.

[3] Game Freak. 2019. Pokémon Sword. Game.

[4] Grezzo. 2019. The Legend of Zelda: Link's Awakening. Game.

[5] Poika Isokoski and Benot Martin. 2006. Eye tracker input in first person shooter games. In Proceedings of the 2nd Conference on Communication by Gaze Interaction: Communication by Gaze Interaction-COGAIN 2006: Gazing into the Future. 78-81.

[6] Robert J. K. Jacob. 1990. What you look at is what you get: eye movement-based interaction techniques. In Proceedings of the SIGCHI conference on Human factors in computing systems: Empowering people (Seattle, Washington, United States) (CHI '90). ACM, New York, NY, USA, 11-18. https://doi.org/10.1145/97243.97246

[7] Pierre Maquet, Jean-Marie Péters, Joël Aerts, Guy Delfiore, Christian Degueldre, André Luxen, and Georges Franck. 1996. Functional neuroanatomy of human rapid-eye-movement sleep and dreaming. Nature 383, 6596 (1996), 163.

[8] Jorge Munoz, Georgios N Yannakakis, Fiona Mulvey, Dan Witzner Hansen, German Gutierrez, and Araceli Sanchis. 2011. Towards gaze-controlled platform 
games. In Computational Intelligence and Games (CIG), 2011 IEEE Conference on. IEEE, 47-54.

[9] Lennart E Nacke, Sophie Stellmach, Dennis Sasse, and Craig A Lindley. 2010. Gameplay experience in a gaze interaction game. arXiv preprint arXiv:1004.0259 (2010).

[10] Joshua Newn, Fraser Allison, Eduardo Velloso, and Frank Vetere. 2018. Looks can be deceiving: Using gaze visualisation to predict and mislead opponents in strategic gameplay. In Proceedings of the 2018 CHI Conference on Human Factors in Computing Systems. ACM, 261.

[11] Argenis Ramirez Gomez and Hans Gellersen. 2019. Exploring the Sensed and Unexpected: Not Looking in Gaze Interaction. In Proceedings of the Halfway to the Future Symposium 2019 (HTTF 2019), November 19-20, 2019, Nottingham, United Kingdom (HTTF 2019). ACM, New York, NY, USA. https://doi.org/10.1145/ 3363384.3363479

[12] Argenis Ramirez Gomez and Hans Gellersen. 2019. Looking Outside the Box: Reflecting on Gaze Interaction in Gameplay. In Proceedings of the 2019 Annual Symposium on Computer-Human Interaction in Play (CHI PLAY '19). ACM, New York, NY, USA. https://doi.org/10.1145/3311350.3347150

[13] Argenis Ramirez Gomez and Hans Gellersen. 2019. SuperVision: Playing with Gaze Aversion and Peripheral Vision. In Proceedings of the 2019 CHI Conference on Human Factors in Computing Systems. ACM, 473.

[14] Argenis Ramirez Gomez and Michael Lankes. 2019. Towards Designing Diegetic Gaze in Games: The Use of Gaze Roles and Metaphors. Multimodal Technologies and Interaction 3, 4 (2019), 65.

[15] C Schaefer, R Menges, K Schmidt, M Kuich, and T Walber. 2014. Schau genau! an eye tracking game with a purpose. Applications for Gaze in Games (2014).

[16] India Starker and Richard A. Bolt. 1990. A Gaze-responsive Self-disclosing Display. In Proceedings of the SIGCHI Conference on Human Factors in Computing Systems (Seattle, Washington, USA) (CHI '90). ACM, New York, NY, USA, 3-10. https://doi.org/10.1145/97243.97245
[17] Tobii. 2016-20. Tobii Gaming Games. https://gaming.tobii.com/games/. Accessed: 2020-03-18.

[18] Eduardo Velloso and Marcus Carter. 2016. The Emergence of EyePlay: A Survey of Eye Interaction in Games. In Proceedings of the 2016 Annual Symposium on Computer-Human Interaction in Play (Austin, Texas, USA) (CHI PLAY'16). ACM, New York, NY, USA, 171-185. https://doi.org/10.1145/2967934.2968084

[19] Stephen Vickers, Howell Istance, and Matthew Smalley. 2010. EyeGuitar: making rhythm based music video games accessible using only eye movements. In Proceedings of the 7th International Conference on Advances in Computer Entertainment Technology. ACM, 36-39.

[20] Melodie Vidal. 2014. Shynosaurs: A Game of Attention Dilemma. In Proceedings of the First ACM SIGCHI Annual Symposium on Computer-human Interaction in Play (Toronto, Ontario, Canada) (CHI PLAY '14). ACM, New York, NY, USA, 391-394. https://doi.org/10.1145/2658537.2662979

[21] Melodie Vidal, Remi Bismuth, Andreas Bulling, and Hans Gellersen. 2015. The Royal Corgi: Exploring Social Gaze Interaction for Immersive Gameplay. In Proceedings of the 33rd Annual ACM Conference on Human Factors in Computing Systems (Seoul, Republic of Korea) (CHI '15). ACM, New York, NY, USA, 115-124. https://doi.org/10.1145/2702123.2702163

[22] Mélodie Vidal, Andreas Bulling, and Hans Gellersen. 2013. Pursuits: Spontaneous Interaction with Displays Based on Smooth Pursuit Eye Movement and Moving Targets. In Proceedings of the 2013 ACM International foint Conference on Pervasive and Ubiquitous Computing (Zurich, Switzerland) (UbiComp '13). ACM, New York, NY, USA, 439-448. https://doi.org/10.1145/2493432.2493477

[23] Tom Wilcox, Mike Evans, Chris Pearce, Nick Pollard, and Veronica Sundstedt. 2008. Gaze and voice based game interaction: the revenge of the killer penguins. SIGGRAPH Posters 81 (2008).

[24] Yanxia Zhang, Andreas Bulling, and Hans Gellersen. 2013. Sideways: A gaze interface for spontaneous interaction with situated displays. In Proceedings of the SIGCHI Conference on Human Factors in Computing Systems. ACM, 851-860. 